\title{
* Introduction to a Systemic Theory of Meaning *
}

\author{
Christophe Menant crmenant[a-t]free.fr
}

\section{1) Introduction.}

a) Information and Meaning are present everywhere around us and within ourselves.

b) Specific studies have been implemented in order to link information and meaning:

- Semiotics

- Phenomenology

- Analytic Philosophy

- Psychology

c) No general coverage is available for the notion of meaning.

d) We propose to complement this lack by a systemic approach to meaning generation.

\section{2) Information and Meaning. Meaning Generator System.}

a) The word "meaning " is most of the time related to the performances of humans.

b) The nature of human is unknown ("Hard Problem") => Nature of "meaning" unknowable.

c) Proposal is to analyse "meaning" at the level of elementary life (paramecium), and reformulate results in a systemic approach with appropriate hypothesis.

d) Definitions and properties of "Meaning" and of "Meaning Generator System" (MGS).

A meaning is a meaningful information that is created by a system submitted to a constraint when it receives an external information that has a connection with the constraint.

The meaning is formed of the connection existing between the received information and the constraint of the system. The function of the meaning is to participate to the determination of an action that will be implemented in order to satisfy the constraint of the system.

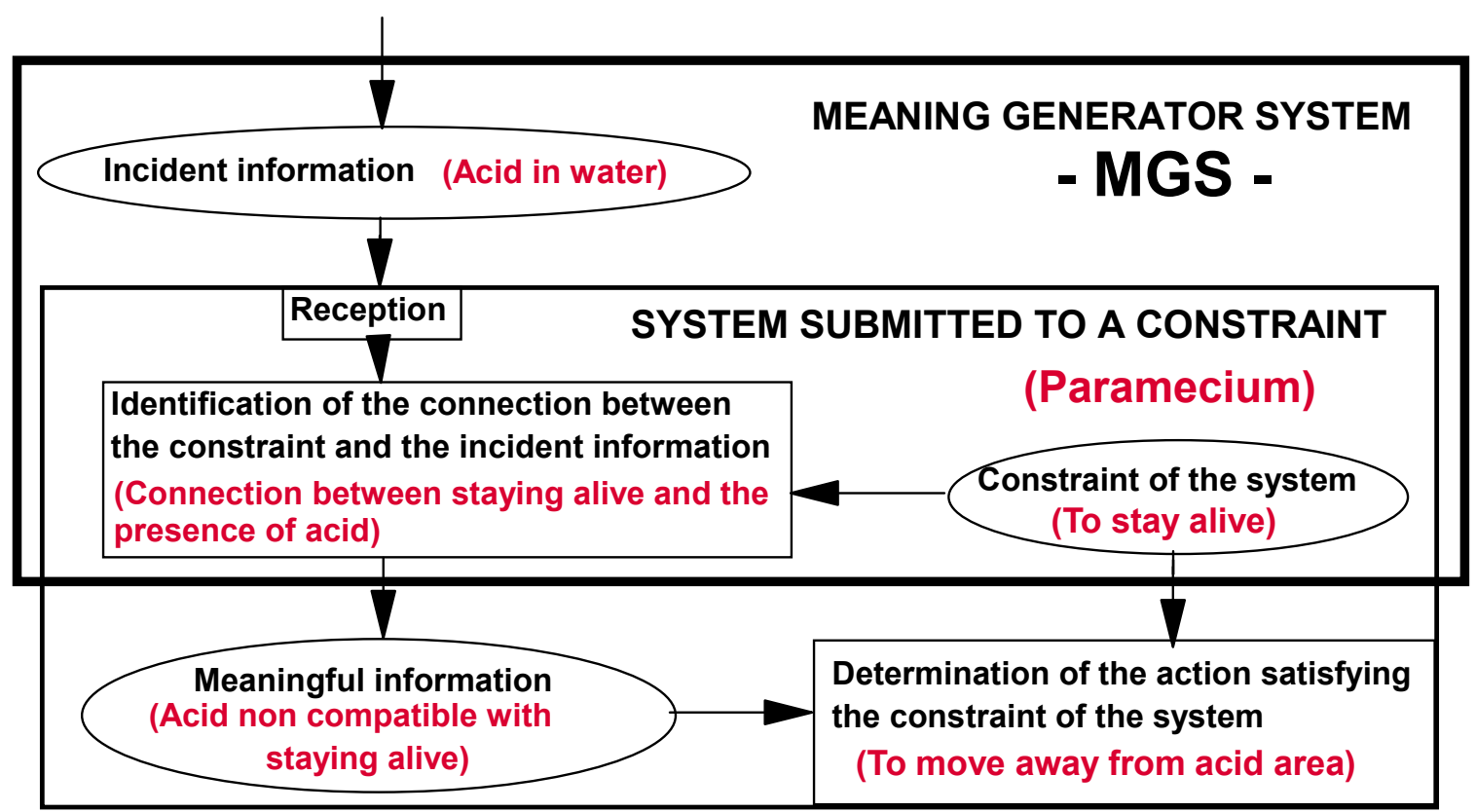




\section{3)MGS Characteristics.}

a) Is a building block, is part of higher level systems.

b) Links characteristics of system (constraint) to the environment (received information).

c) Uses different types of constraints for different levels of evolution.

d) Allows meaningful information groundings "in" and "out" of the system that produced it.

e) Introduces possibilities for evolutionary groundings.

f) Several MGSs can be related to build up networks of meanings.

g) Needs some conceptualization of the notion of Constraint.

\section{4) Transmission of a Meaning.}

a) Meaningful Information can be transmitted and survive to MGS.

b) Introduction of "Efficiency of Meaning" and of "Domain of Efficiency of a Meaning".

\section{5) Conclusion and Continuation.}

a) Basic elements for a Systemic Theory of Meaning have been introduced.

b) Key notions have been defined, with corresponding properties (Meaning, Constraint, MGS, Groundings in/out, Meaning Transmission, Efficiency of a Meaning).

c) Continuation with application to specific domains (life, human, robots) is in process.

(See Biosemiotics 2003, TSC 2004, FIS 2005).

d) Presentations and publication on subject are available at $\underline{\text { Home Page }}$ 